\title{
The ATLAS Muon Spectrometer
}

\author{
L. Pontecorvo ${ }^{1,2}$ \\ On behalf of the ATLAS Collaboration \\ 1 CERN \\ 2 INFN Roma; \\ e-mail: Ludovico.pontecorvo@roma1.infn.it
}

\begin{abstract}
This paper presents the Muon Spectrometer of the ATLAS detector. The general characteristics of this system as acceptance, resolution and trigger capabilities are discussed together with the main physics goals and the background conditions. Four different detector technologies are used for triggering and precise tracking in the spectrometer; in the following, a description of the main characteristics of each of them, focusing on test beam results, is presented.
\end{abstract}

PACS: not given

\section{Introduction}

The ATLAS Collaboration is building a general purpose detector to study protonproton interaction at $14 \mathrm{TeV}$ at the Large Hadron Collider (LHC) at CERN [1]. This paper deals with the Muon Spectrometer which is based on a system of three large superconducting air-core toroids. Figure 1 shows the general ATLAS layout. The detector size is about $22 \mathrm{~m}$ in diameter and $44 \mathrm{~m}$ in length.

The design of the Muon Spectrometer was driven by the need of having a very high quality stand-alone muon measurement, with large acceptance both for muon triggering and measuring, to achieve the physics goals discussed in section 3. Precision tracking in the Muon Spectrometer is achieved using high precision drift and multiwire proportional chambers. Great emphasis has been given in the design phase to system issues such as the alignment of the tracking detectors. Triggering is accomplished using dedicated fast detectors, that allow bunch crossing identification, with limited spatial accuracy. These detectors provide also the measurement of the coordinate in the non-bending plane.

In the following sections we shall review the Muon Spectrometer design, the experiment's physics goals, the muon trigger system and the chamber technologies involved and the tracking system with its different chambers. Finally some conclusions are drawn.

\section{The Muon Spectrometer}

The conceptual design of the Muon Spectrometer [2] is illustrated in Figure 2.

The spectrometer is divided into three regions: a Barrel extending in the rapidity region $|\eta| \leq 1.2$ and two End-Caps covering the rapidity regions $(1<$ 


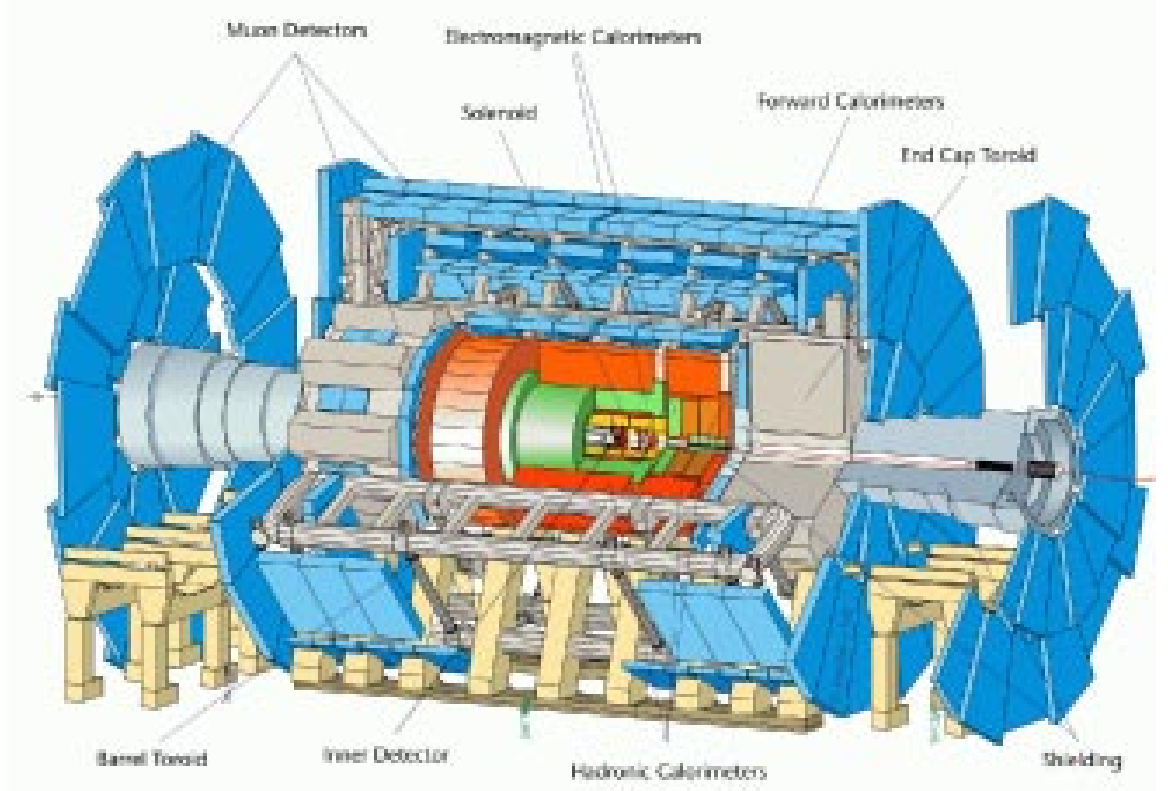

Fig. 1. Layout of the ATLAS Detector.

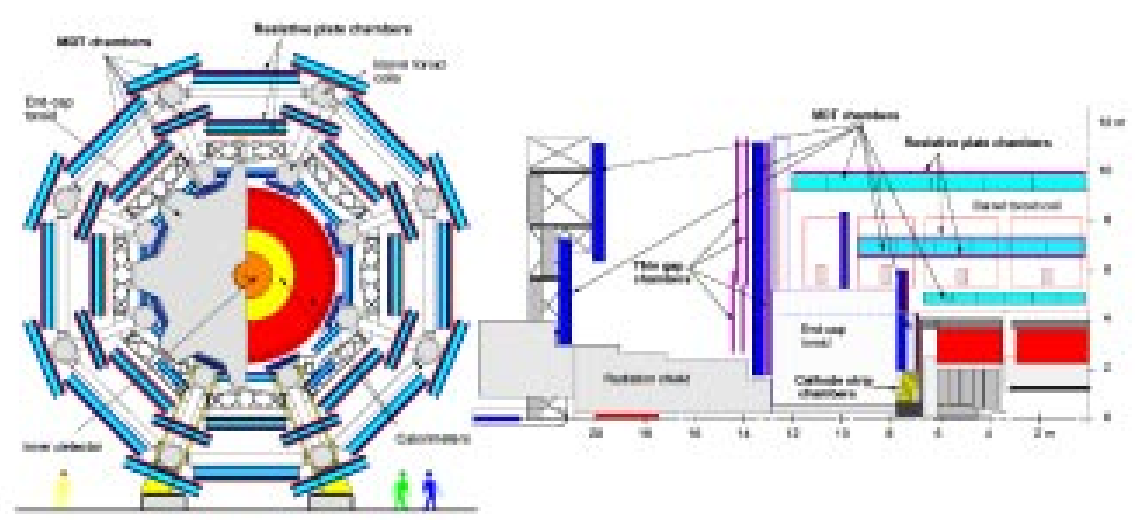

Fig. 2. Conceptual design of the muon spectrometer.

$|\eta|<2.7)$. In the Barrel, the toroidal field is produced by eight very large superconducting coils arranged in an open geometry. The field integral in the Barrel varies between 2 and $5 \mathrm{Tm}$, with large variation as a function of the azimuth angle. The muon momentum measurement is obtained measuring the saggitta of the muon trajectory produced by the magnetic field. The muon trajectory is sampled in three high precision measuring stations placed inside the toroid, equipped with Monitored Drift Tubes (MDT - see section 5) and arranged in 
three cylindrical layers around the beam axis. Each station measures the muon positions with a precision of about $50 \mu \mathrm{m}$. It also provides angular information on the measured track segments, which is then used to improve the pattern recognition for the reconstruction of the full muon track. In the two outer stations of the Barrel spectrometer, specialized trigger detectors (Resistive Plate Chambers - RPC- see section 4.2) are present. In the middle station two layers, each comprising two RPC detectors, are used to form a low $\mathrm{p}_{t}$ trigger $\mathrm{p}_{t}>6$ $\mathrm{GeV} / \mathrm{c}$ ). In the outer station only one layer with two RPC detectors is used to form the high $\mathrm{p}_{t}$ trigger $\left(\mathrm{p}_{t}>20 \mathrm{GeV} / \mathrm{c}\right)$, together with the low $\mathrm{p}_{t}$ station. The RPCs measure both the bending and the non-bending coordinate, and the trigger formation requires fast $(<25 \mathrm{~ns})$ coincidences pointing to the interaction region both in the bending and in the non-bending planes.

In the End-Cap regions, two identical air core toroids are placed inside the barrel toroid with the same axis (corresponding to the beam direction). The measurement of the muon momentum is accomplished using three measuring stations of chambers mounted to form three big disks called wheels that are normal to the beam direction, and measuring the angular displacement of the muon track when passing in the magnetic field (the toroids are placed between the first and the second tracking stations). In this case the toroids' volume is not instrumented: a sagitta measurement is not possible hence a point-angle measurement is performed. MDT chambers are used for precise tracking in the full angular acceptance, with the exception of the inner station where the region $2<|\eta|<2.7$ is equipped with Cathode Strip Chambers (CSC see section 5.2) which exhibit a smaller occupancy. The CSCs have a spatial resolution in the range of $50 \mu \mathrm{m}$.

The trigger acceptance in the End-Cap is limited to $(|\eta|<2.4)$ where Thin Gap Chambers (TGC see section 4.3) are used to provide the trigger. The TGCs are arranged in two stations: one made of two doublets of two layers each, used for the low $\mathrm{p}_{t}$ trigger, and one made of three layers used for the high $\mathrm{p}_{t}$ trigger in conjunction with the low $\mathrm{p}_{t}$ stations. The high $\mathrm{p}_{t}$ station is placed in front of the middle precision tracking wheel and the low $\mathrm{p}_{t}$ station is behind it. The TGCs provide also the measurement of the second coordinate and for this reason there is a TGC layer also in the first tracking wheel.

The design of the Muon Spectrometer has been optimized for high standalone resolution and robust muon identification.

Figure $3 \mathrm{~A}$ presents the stand-alone momentum resolution as a function of muon momentum together with the various contributions that limit the resolution in the different momentum ranges. For momenta below $10 \mathrm{GeV} / \mathrm{c}$, the fluctuation on the energy loss of muons in the calorimeters limit the resolution to about $6-8 \%$. For momenta up to $250 \mathrm{GeV} / \mathrm{c}$ the multiple scattering in the materials present in the spectrometer limit the resolution to about $2 \%$, while for higher momenta the spatial accuracy of the chambers and the knowledge of their calibration and alignment give the largest contribution to the resolution. As an example, a $1 \mathrm{TeV} / \mathrm{c}$ momentum muon is measured with a $10 \%$ resolution. To improve the muon measurement resolution at low momenta (below $100 \mathrm{GeV} / \mathrm{c}$ ) it is possible to use a combined reconstruction of the muon trajectory using also the Inner Tracker. In this case the Muon Spectrometer is used mainly for the 

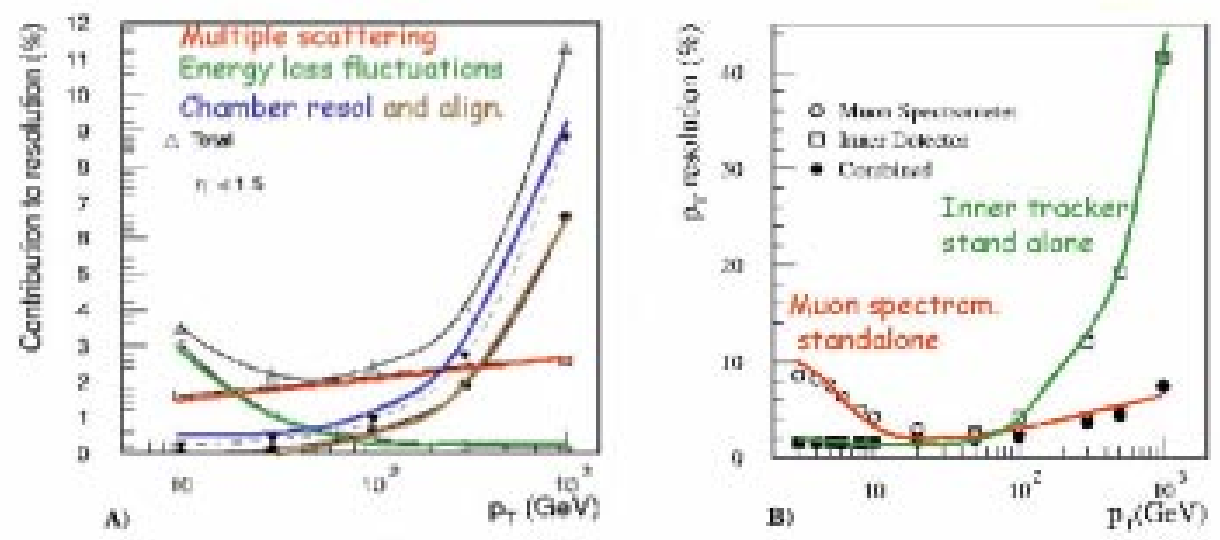

Fig. 3. A) Resolution as a function of the muon momentum for the stand-alone Muon Spectrometer. Also displayed are the different contribution to the resolution. B) Resolution as a function of the muon momentum for the stand-alone Muon Spectrometer, stand-alone Inner Tracker and combined measurement.

identification of the muon. Figure 3B shows the stand-alone resolution for the Inner Tracker and the Muon Spectrometer, together with the combined one. In ATLAS a muon is measured with a precision of about $2 \%$ for momenta up to $250 \mathrm{GeV} / \mathrm{c}$.

\section{Physics goals and backgrounds}

Experiments at the LHC have a very rich physics potential: for example, the discovery of new particles e.g. Higgs bosons, the discovery of a large variety of new supersymmetric particles, high accuracy measurements of the mass and couplings of gauge bosons and quarks as well as the production of new heavy gauge bosons ( $W^{\prime}$ and $Z^{\prime}$ ), and accurate studies of the $\mathrm{CP}$ violation in the Beauty sector [3]. Most of these processes imply the presence of muons in the final states and the ATLAS Muon Spectrometer is an essential device for enhancing the discovery potential of the experiment. The momentum range spanned by the muons produced in interesting reactions is very large, going from a few $\mathrm{GeV} / \mathrm{c}$ for the muons produced in Beauty decays to the $\mathrm{TeV} / \mathrm{c}$ range for muons produced in new heavy gauge bosons decays. The Muon Spectrometer performance have been optimized using as benchmarks the following reactions:

1. SM Higgs decays in the full mass range accessible to LHC

2. MSSM Higgs decays with particular attention to the $A \rightarrow \mu \mu$ decays

3. New vector boson decays for boson masses up to $3-4 \mathrm{TeV} / \mathrm{c}^{2}$

4. Beauty decays 
For the Standard Model Higgs search, different final states are relevant as a function of the Higgs mass. In the $130<M_{H}<170 \mathrm{GeV} / \mathrm{c}^{2}$ mass range the Muon Spectrometer is mainly used to trigger and measure the muons coming from the $H \rightarrow Z Z^{*}$ and $H \rightarrow W W^{*}$. The typical momentum range of such muons is $5<\mathrm{p}_{t}<50 \mathrm{GeV} / \mathrm{c}$ and the momentum resolution of the spectrometer is limited to $2-8 \%$ by energy loss fluctuation in the calorimeters and by multiple scattering. Figure 4 shows how a $130 \mathrm{GeV} / \mathrm{c}^{2}$ Higgs decaying into four muons would be reconstructed in ATLAS using only the Muon Spectrometer.

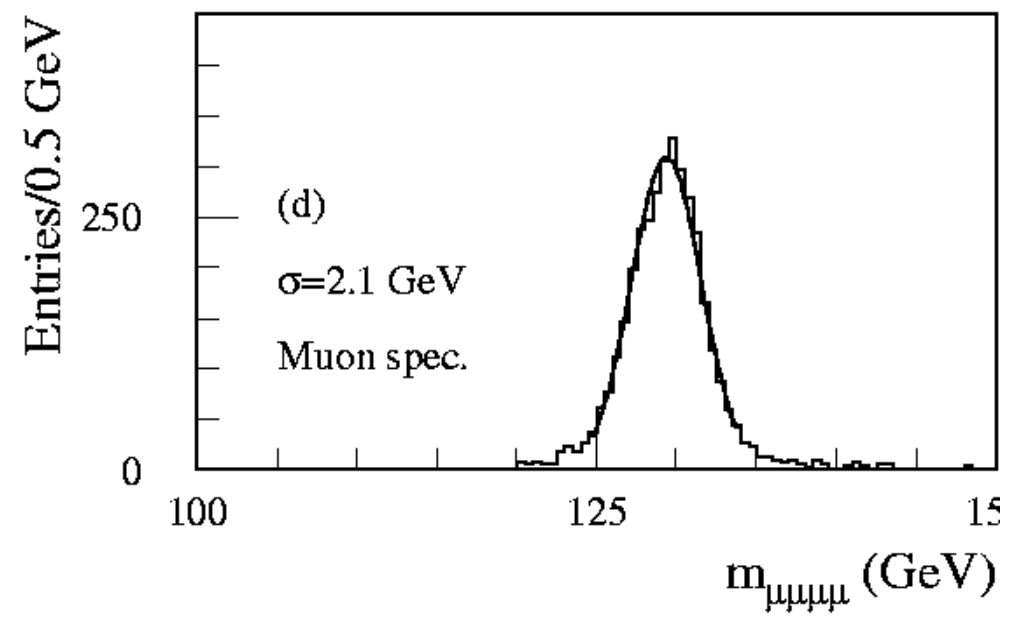

Fig. 4. Reconstruction of a $130 \mathrm{GeV} / c^{2}$ Higgs using only the Muon Spectrometer.

The width of the mass peak is about $2.1 \mathrm{GeV} / \mathrm{c}^{2}$ which is improved to about $1.4 \mathrm{GeV} / \mathrm{c}^{2}$ using also the inner tracker measurement. For higher Higgs masses, the golden channel for the search is $H \rightarrow Z Z$, in which the Z's decay into leptons (muons and/or electrons). In this case the muons have large momenta and the measurement performed in the Muon Spectrometer is superior to the one performed in the inner tracker. An interesting channel where the high resolution of the Muon Spectrometer plays a crucial role in the discovery potential of the ATLAS experiment is the MSSM Higgs A boson decaying into two muons. This is a rather narrow state even for high $\mathrm{A}$ mass $\left(M_{A}<300 \mathrm{GeV} / \mathrm{c}^{2}\right)$. The decay channel involving muons could be very useful in the precise determination of the A mass (compared with the $A \rightarrow \tau \tau$ channel), and a measurement of the coupling of the A to $\tau$ 's and muons could be performed. Fig 5 shows the region of the plane $\mathrm{M}_{A} \operatorname{Tan} \beta$ accessible to ATLAS.

The main source of background in the Muon Spectrometer is a large number of photons and neutrons, with energies typically below $1 \mathrm{MeV}$ and $100 \mathrm{keV}$ respectively, which interact with low probability in the active volume of the tracking and trigger detectors. Typical rates in the Barrel sector are below 20 $\mathrm{Hz} / \mathrm{cm}^{2}$ for almost all the chambers, but it increases fast with rapidity: rates of the order of $1 \mathrm{kHz} / \mathrm{cm}^{2}$ are foreseen at the largest rapidity. Fig $6 \mathrm{~A}$ shows the 


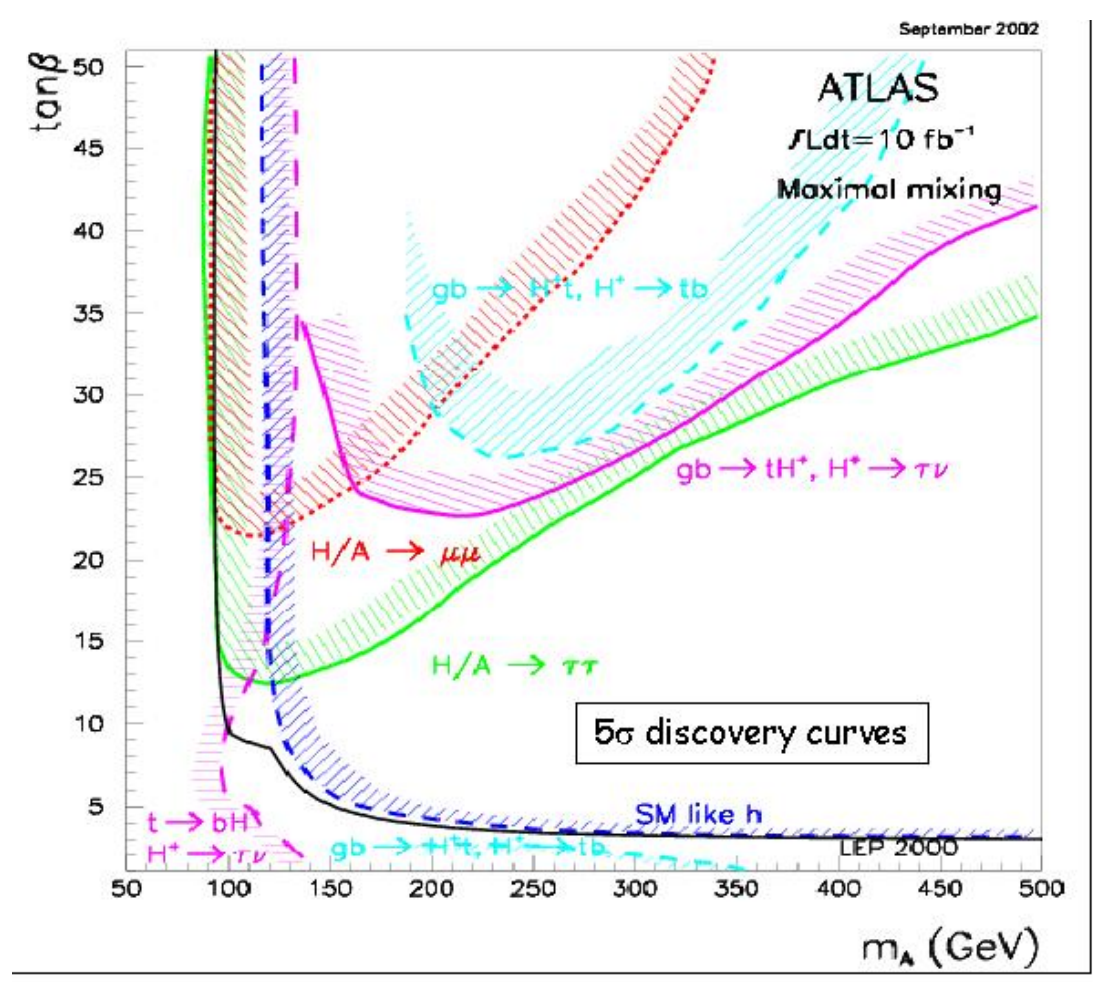

Fig. 5. 5 sigma discovery curves in the plane $\mathrm{M}_{A}$ Tan $\beta$ for MSSM Higgs particles, using different decay channels.

counting rate as a function of $\eta$ on all the three stations of the detector.

The rate due to muons depends strongly on the threshold on transverse momentum. At a luminosity of $10^{34} \mathrm{~cm}^{-2} \mathrm{~s}^{-1}$ the total rate for $\mathrm{p}_{t}>8 \mathrm{GeV} / \mathrm{c}$ is $\simeq 30 \mathrm{kHz}$, reducing to $\simeq 1 \mathrm{kHz}$ above $20 \mathrm{GeV} / \mathrm{c}$. In this range, as illustrated in Fig 6B, muon production is dominated by light mesons decays, Beauty and Charm decays and further above by $\mathrm{W}$ decays.

\section{The muon trigger}

The physics program outlined above demands for a highly flexible trigger scheme with different programmable transverse momentum thresholds. At low luminosity a $6 \mathrm{GeV} / \mathrm{c}$ threshold for two or more muons is adequate for Beauty physics, while higher transverse momentum thresholds $(\mathrm{eg} 20 \mathrm{GeV} / \mathrm{c})$ will be used for Higgs search and high $\mathrm{p}_{t}$ physics measurements. The muon trigger in ATLAS is organized in three levels. The Level-1 trigger (LVL1) uses reduced-granularity data, coming only from the trigger detectors. The second level (LVL2) uses full granularity and precision data from most of the detectors, but examines only the regions of the detector flagged at the LVL1 as containing interesting information. 

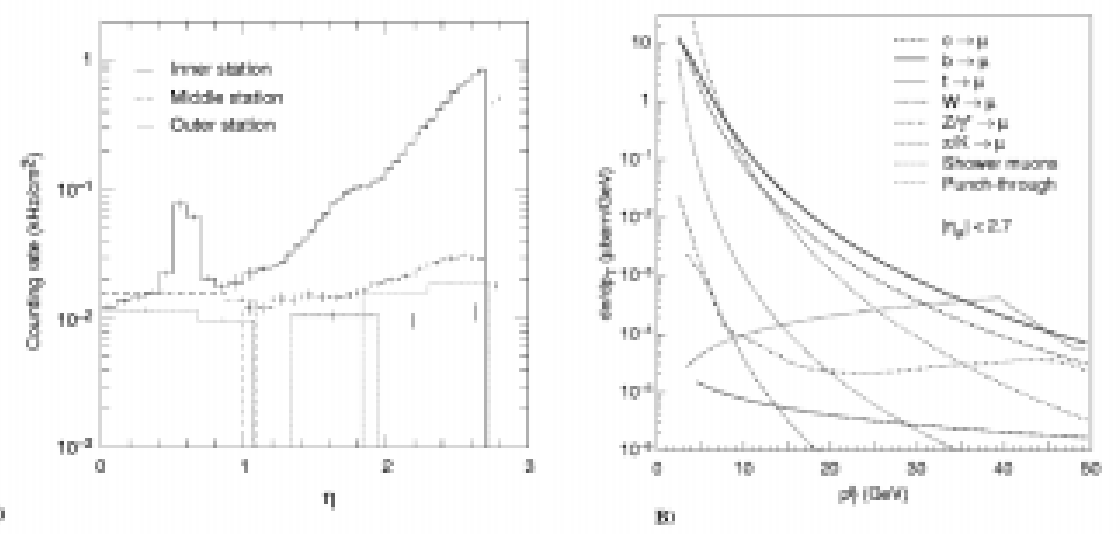

Fig. 6. A)Expected counting rate due to photons and neutrons as a function of the rapidity in the three measuring stations. B) Muon rate in $\mu$ barn $/ \mathrm{GeV}$ as a function of the transverse momentum integrated over $|\eta|<2.7$.

The Level 3 trigger (LVL3) uses the full event data for final selection of events, applying the offline reconstruction. Typical rates at the three trigger levels are $75 \mathrm{kHz}$ (LVL1), $1 \mathrm{kHz}$ (LVL2) and $100 \mathrm{~Hz}$ (LVL3).

\subsection{Level-1 trigger.}

The Level-1 trigger uses specialized trigger detectors: RPCs in the Barrel and TGCs in the End-Cap. Both these chambers have enough time resolution to correctly identify the bunch crossing, which is one of the main tasks of the first level trigger. The trigger scheme used in both the Barrel and the End-Cap is illustrated in Fig. 7.

Three layers of detectors are used. In the Barrel the low $\mathrm{p}_{t}$ trigger uses predefined coincidence patterns, in both projections, using the middle station RPCs. The momentum resolution is about $20 \%$ and is limited mainly by multiple scattering and by fluctuation of the energy loss in the calorimeters. The high $\mathrm{p}_{t}$ trigger requires a coincidence pattern using all three RPC stations. At $\mathrm{p}_{t}$ of 20 $\mathrm{GeV} / \mathrm{c}$ the momentum resolution is about $30 \%$ and is limited by the axial length of the interaction region and by multiple scattering in the central calorimeters. The same logic is applied to the trigger scheme in the End-Cap. The $\mathrm{p}_{t}$ threshold is defined by the width of the coincidence patterns and can be programmed. This width depends on the rapidity, and for a $20 \mathrm{GeV} / \mathrm{c}$ threshold it varies from about $40 \mathrm{~cm}$ in the Barrel to about $5 \mathrm{~cm}$ in the End-Cap. The efficiency curves for this trigger scheme both for low and high $\mathrm{p}_{t}$ are presented in Fig 8

\subsection{The Resistive Plate Chambers: RPC.}

The RPCs are gaseous detectors made of two parallel resistive bakelite plates separated by insulating spacers which form a $2 \mathrm{~mm}$ gas gap [4]. High voltage is 


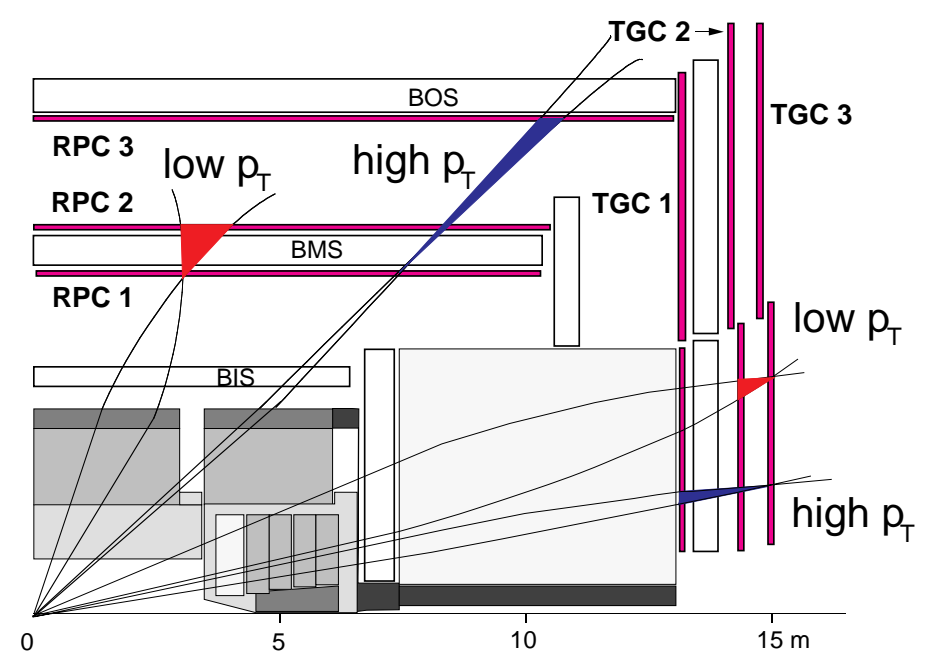

Fig. 7. Trigger scheme for high and low $\mathrm{p}_{t}$ thresholds in the Barrel and in the End-Cap.

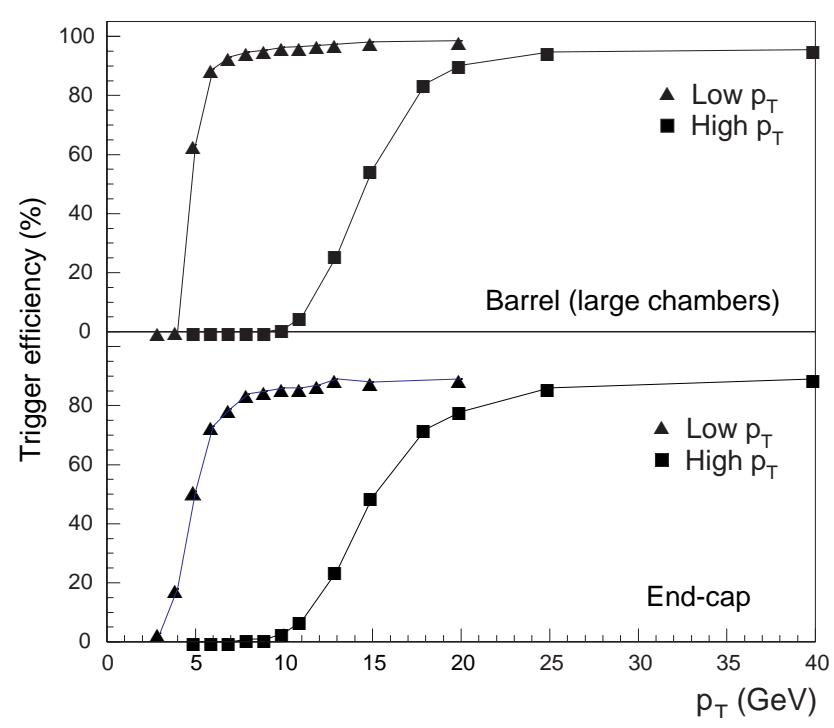

Fig. 8. Trigger efficiency both for high and low $\mathrm{p}_{t}$ trigger in the Barrel and in the End-Cap.

applied on these plates through graphite electrodes, and electrons produced by an ionizing particle in the gas gap are multiplied into avalanches. The electrical signals produced in the gas gap are induced on two read out copper strip planes placed on both sides of the gap, and then amplified and discriminated by fast amplifiers. Fig 9 presents a schematic view of an RPC.

The gas used is: $\mathrm{C}_{2} \mathrm{H}_{2} \mathrm{~F}_{4} 94.7 \%, \mathrm{C}_{4} \mathrm{H}_{10} 5 \%$ and $S F_{6} 0.3 \%$, resulting in a non- 


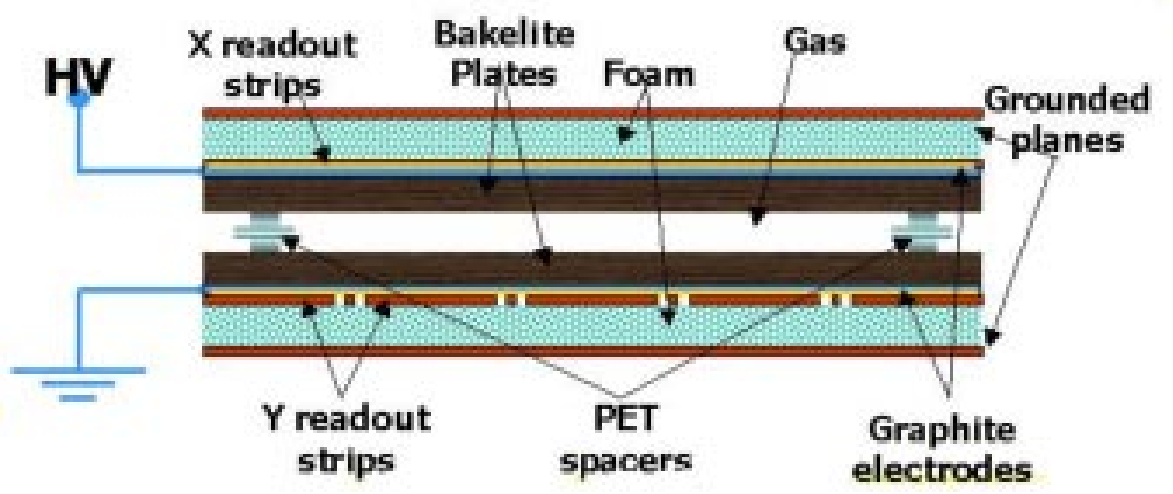

Fig. 9. Schematic view of an RPC.

flammable environmentally safe gas mixture. $S F_{6}$ is used to limit the charge produced in each pulse and the streamer probability. A typical value for the electric field is $5.0 \mathrm{kV} / \mathrm{mm}$ and for the avalanche charge, $0.5 \mathrm{pC}$. Typical spacetime resolutions of $1 \mathrm{~cm} \times 1 \mathrm{~ns}$ are achieved.

The RPC chambers used in ATLAS are made of two gas volumes, each of which is read-out by two orthogonal strip planes. Each chamber is tested in a Cosmic Ray Stand after production, where measurements of the voltage-current characteristic, noise, efficiency plateau are performed, as well as a "muon radiography" to search for any possible inefficient zone of the detector. Typical results of such tests are presented in Fig 10 and Fig 11. The efficiency plateau is approaching $98 \%$ and from the "muon radiography" it is evident that the inefficiencies are concentrated only in the spacers' positions.

\subsection{The Thin Gap Chambers: TGC.}

The Thin Gap Chambers are multiwire proportional chambers with a smaller distance between the cathode and the wire plane compared with the distance between wires (Fig 12) [5].

The main dimensional characteristics of the chambers are a cathode distance of $2.8 \mathrm{~mm}$, a wire pitch of $1.8 \mathrm{~mm}$ and a wire diameter of $50 \mu \mathrm{m}$. The gas mixture is $55 \% \mathrm{CO}_{2}$ and $45 \% \mathrm{n}$-pentane, which results in a highly quenching gas mixture that permits the operation in saturated avalanche mode. This mode of operation enables a small sensitivity to mechanical deformations, a small dependence of the pulse height on the crossing angle and a very small streamer formation probability. The chambers are operated at a high voltage of about $3 \mathrm{kV}$. The operating condition and the electric field configuration provide for a short drift time, enabling a good time resolution of about $4 \mathrm{~ns}$. The read-out of the signals is done both from the wires (which are grouped together in variable numbers between 4 and 40, according to the desired trigger granularity as a function of the pseudorapidity) and from a pick-up strip plane placed on the cathode. The wire planes and the strips are perpendicular to each other anabling the measurement 


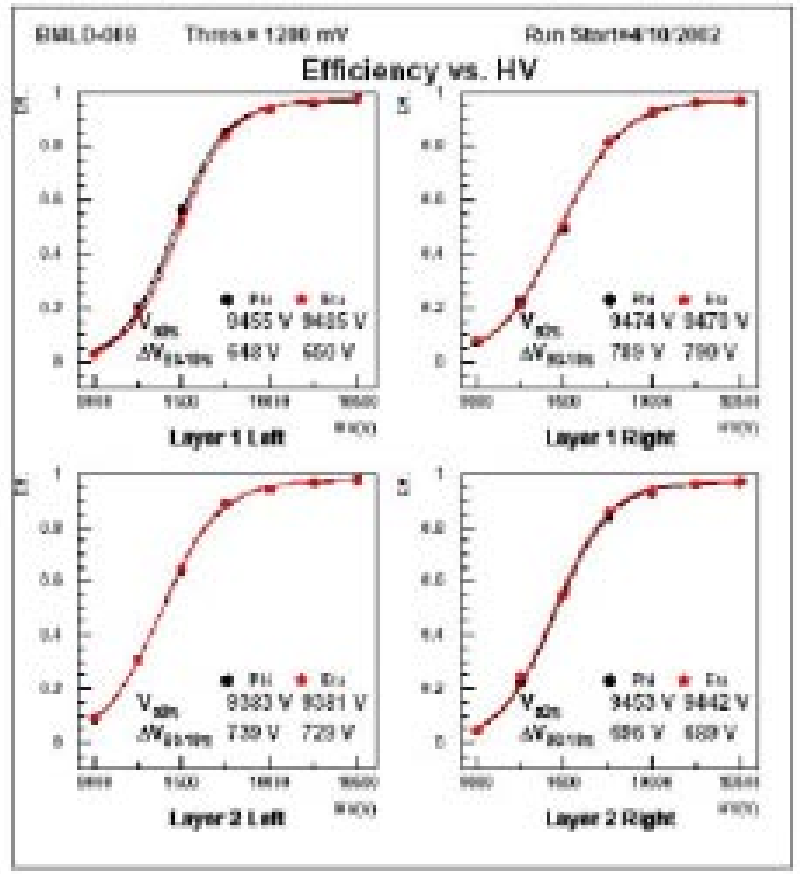

Fig. 10. Efficiency Plateau

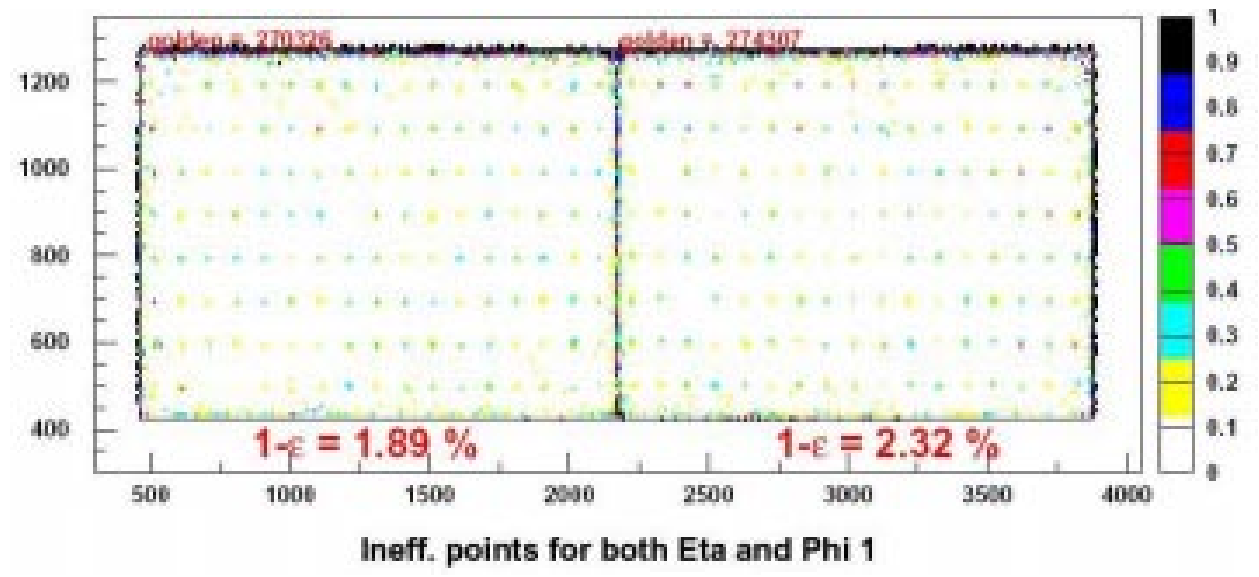

Fig. 11. Muon radiography, colored spots indicate inefficiency and are concentrated at the spacer locations.

of two orthogonal coordinates, only the wire signals are used in the trigger logic.

The TGCs chambers have been chosen for the End-Cap of the ATLAS Muon Spectrometer because of their very good rate capability and ageing character- 


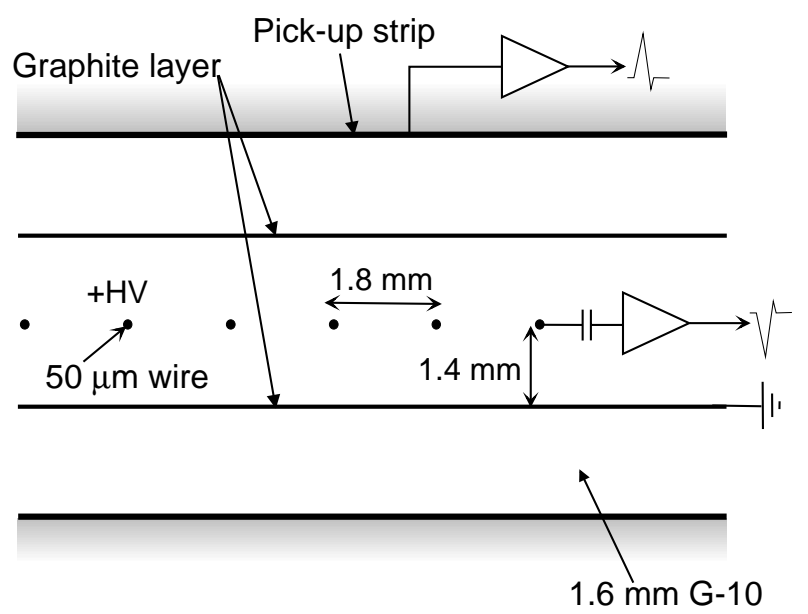

Fig. 12. Schematic view of a TGC.

istics. Tests have been made at the CERN Gamma Irradiation Facility (GIF), where a $740 \mathrm{GBq}{ }^{137} \mathrm{Cs}$ source is used to irradiate the chambers. The typical counting rate at which the tests have been performed was $1 \mathrm{kHz} / \mathrm{cm}^{2}$. The efficiency and the time resolution of the chambers were measured by exposing the TGC chambers to a muon beam and the results are presented in Fig 13.
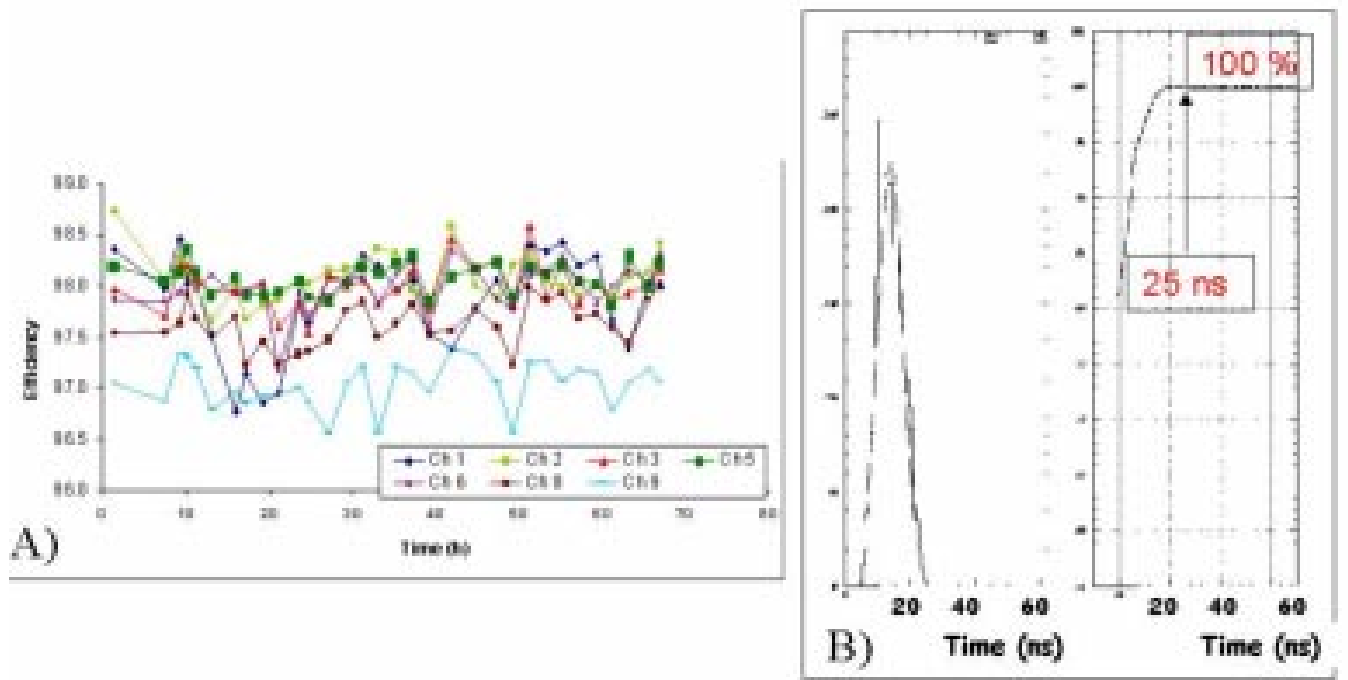

Fig. 13. Efficiency under irradiation $\left(1 \mathrm{kHz} / \mathrm{cm}^{2}\right)$ for 7 chambers under test at GIF. B) Time distribution for the TGC hits, and percentage of TGC hits found as a function of a time window. 
The efficiency of the chambers under irradiation is about $98 \%$ - very close to the one measured without irradiation. The time distribution of the hits is all contained in a $25 \mathrm{~ns}$ window demonstrating the feasibility of identifying the bunch crossing at LHC using TGCs. Also the trigger algorithm has been tested (offline) using the muon beam and the source irradiation, and a trigger efficiency of about $99.9 \%$ has been achieved.

\section{The Tracking Chambers}

\subsection{The Monitored Drift Tubes: MDT.}

The precision tracking in ATLAS is performed in almost all the spectrometer by the Monitored Drift Tubes chambers. The basic element for these chambers is a thin walled $(400 \mu \mathrm{m})$ aluminum tube of a diameter of $3 \mathrm{~cm}$ with a length varying from 0.9 to $6.2 \mathrm{~m}$, in which a $50 \mu \mathrm{m} \mathrm{W}$-Rn wire is strung with high mechanical precision. These tubes are assembled in two multilayers which are kept separated by three cross-plates, as shown in Fig. 14.

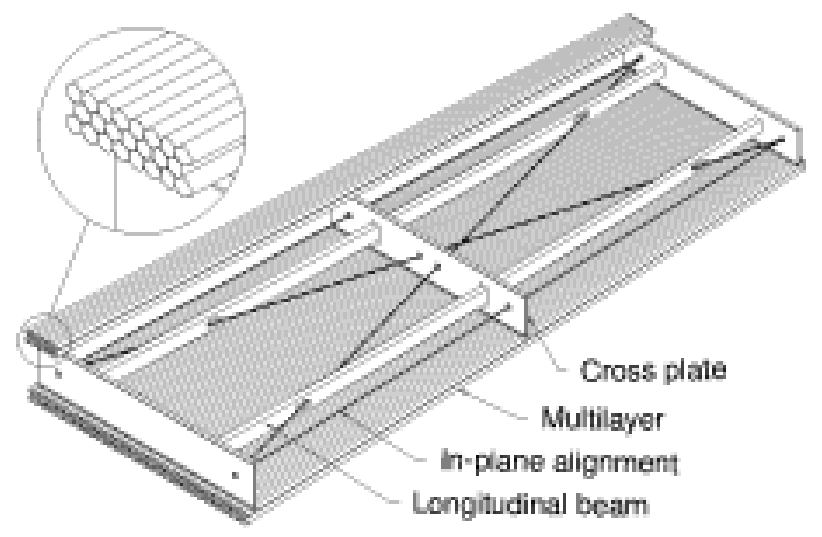

Fig. 14. Scheme of a Monitored Drift Tube chamber.

The multilayers are formed by 3 or 4 layers of tubes, four-layer chambers being used in the inner stations. The mechanical accuracy in the construction of these chambers is extremely tight to meet the momentum resolution requirements of the spectrometer. The precision in wire positions inside a chamber should be higher than $20 \mu \mathrm{m}$ r.m.s.; this has been checked on the first chambers produced using an X-Ray Tomograph [8] which measure the wire position inside a chamber with an accuracy of less than $5 \mu \mathrm{m}$. The required high $\mathrm{p}_{t}$ resolution crucially depends also on the single tube resolution, defined by the operating point, the accurate knowledge of the calibration ( $r-t$ relation) and of the chambers' alignment.

The chambers are filled with a gas mixture of $\mathrm{Ar} 93 \%$ and $\mathrm{CO}_{2} 7 \%$, at 3 bar absolute pressure, and are operated at a gas gain of $2 \times 10^{4}$ applying $3.1 \mathrm{kV}$ on the wires. High pressure ensures high spatial resolution up to large drift radii. 
The resolution curve has been measured as a function of the drift distance under different irradiation conditions at the GIF. The results shown in Fig. 15 indicate that when the chamber is not irradiated an average resolution of about $80 \mu \mathrm{m}$ per wire is obtained.

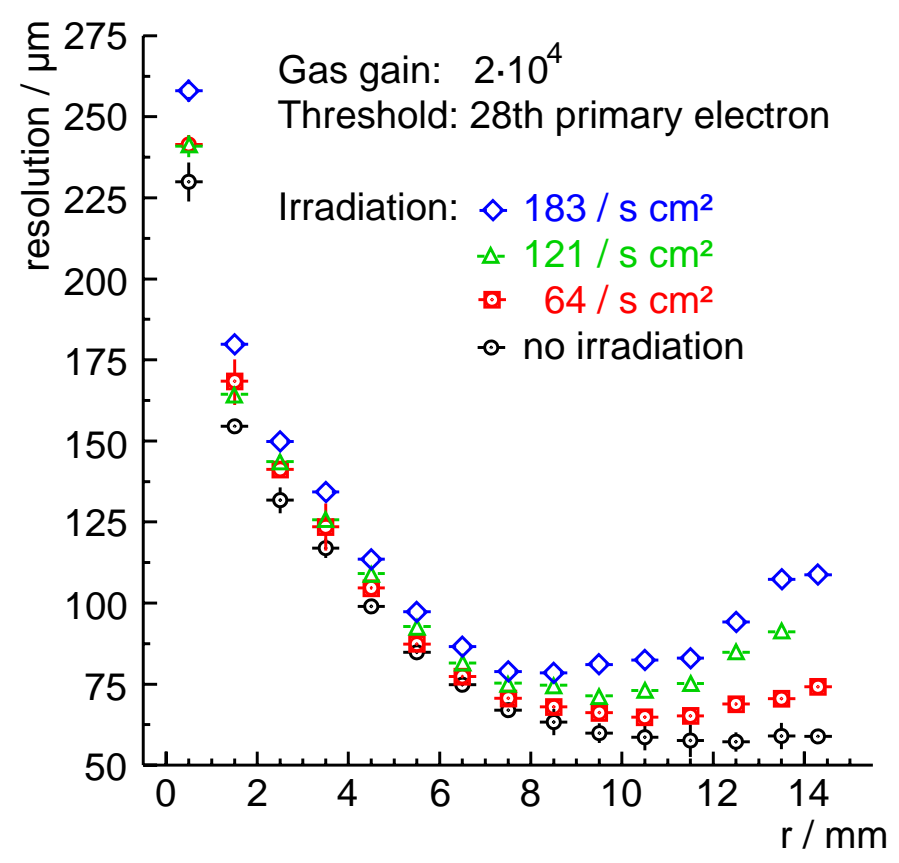

Fig. 15. Single wire resolution as a function of the drift distance for different counting rates.

The resolution degrades at large distances from the wire when the counting rate increases. This is due to space-charge effects that produce fluctuations of the average field seen by an electron drifting to the wire, changing the r-t relation on event basis.

The RT-relation of the tubes in ATLAS should be measured using only information coming from the MDTs. An autocalibration procedure [9],[10] has been developed to obtain an $\mathrm{r}$-t relation which differs from the actual one by less than $30 \mu \mathrm{m}$ over almost all the drift distance. This procedure has been tested using test beam data, comparing the results of the procedure with the r-t calibration obtained using an external tracker. Fig. 16 presents the difference between the autocalibrated $\mathrm{r}$-t relation and the one from the external tracker. Over the full drift-distance the difference is less than $25 \mu \mathrm{m}$, except close to the wire, where threshold effects distort the r-t relation.

The last ingredient for achieving the desired resolution at high transverse momentum is an excellent alignment system that enables the monitoring of the position of the different chambers in the spectrometer with a precision higher than $30 \mu \mathrm{m}[6]$. The basic elements of this system are RASNIK [7] optical straightness monitors, formed by three elements along a view line: a laser that illuminates a 


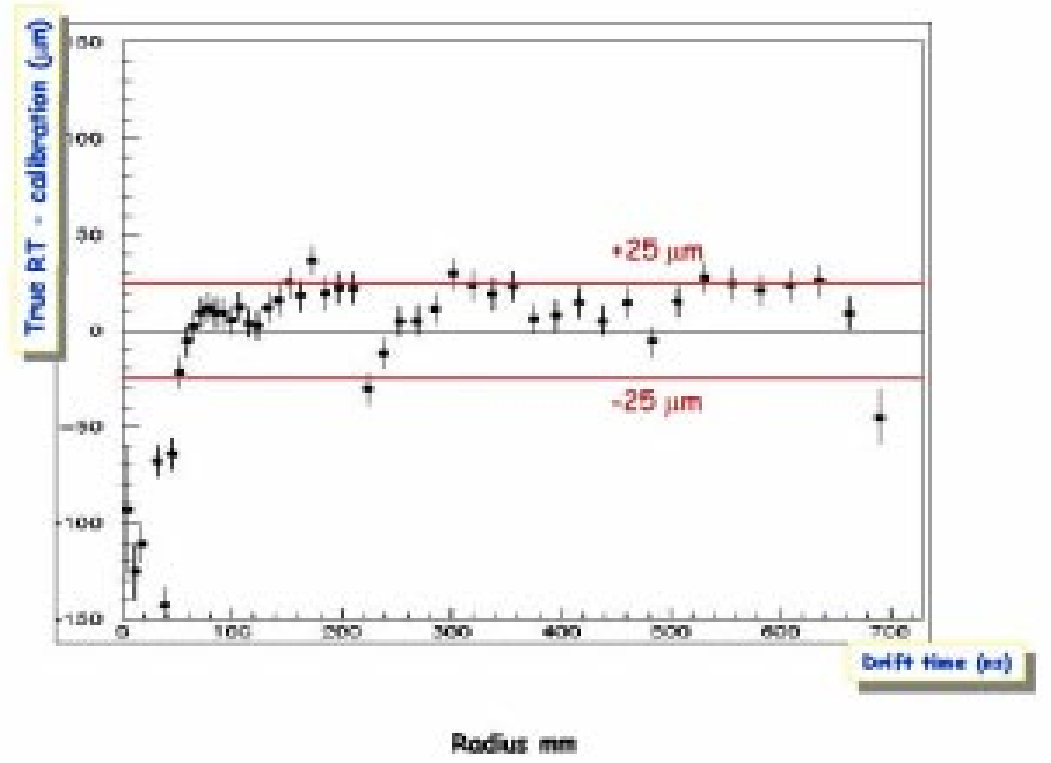

Fig. 16. Difference between autocalibrated r-t relation and external tracker calibration.

coded target mask at one end, a lens in the middle and a CCD sensor at the other end. This system provides a very accurate measurement of the relative alignment of three objects ( $1 \mu \mathrm{m}$ r.m.s.) and is used both for checking the in-chamber deformations (in-plane alignment), and the relative displacement of different chambers (axial-praxial and projective alignment). The in-plane alignment measures the deformation of the chamber cross-plates in which 2-4 RASNIK lines are integrated. These deformations could be due to the effect of gravitation or other strain forces, or temperature gradients along the chamber. The projective system measures the displacements of a chamber in the middle tracking station with respect to the other two stations, making use of RASNIK lines mounted on platforms glued on the chamber surface and pointing back to the interaction region. Since not all the chambers have the projective system, the position of the other chambers within a sector of a station is inferred making use of axial RASNIK lines that connect adjacent chambers. In the End-Cap region, the use of many projective lines is not possible because of the presence of the cryostat of the toroid. Hence, the alignment is performed using a mesh of eight alignment bars per tracking station. These bars are equipped with alignment devices called BCAM that monitor the position of each bar with respect both to the adjacent ones in the same station and to the corresponding ones in the other stations, along two or three projective lines. Moreover the bars have three RASNIK systems inside, with which the deformation of the bar itself is monitored. Once the position of the bars in the mesh is determined, the position of the chambers is then inferred using a set of proximity sensors. These are RASNIK devices 
mounted on the chambers and on the alignment bars, measuring the position of the chamber with respect to the alignment bar. Fig. 17 shows the conceptual design of the alignment system in the Barrel.

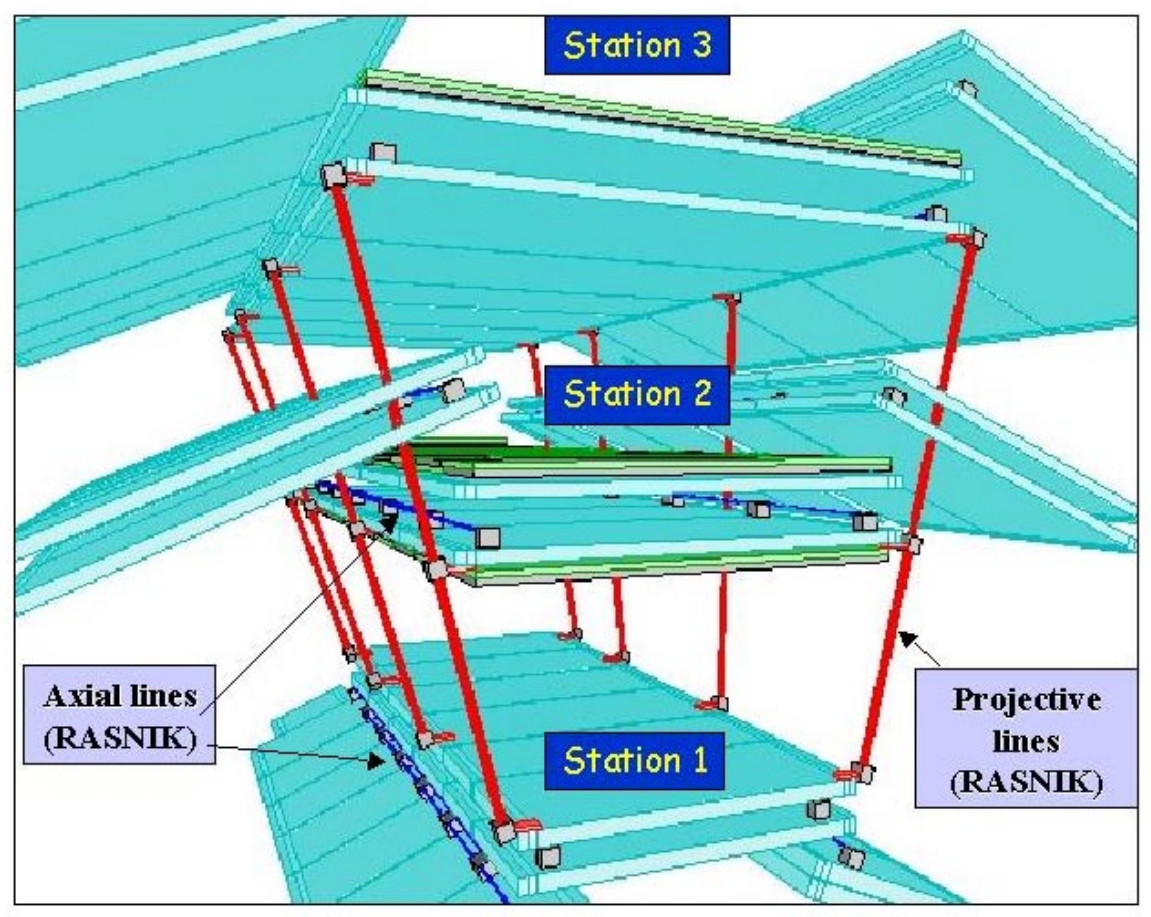

Fig. 17. Conceptual design of the alignment system in the Barrel. Projective and axial lines are displayed.

The accuracy of the Barrel and End-Cap alignment system has been tested during the 2002 ATLAS Muon System Test at the H8 beam line at CERN, using muons of $20 \mathrm{GeV} / \mathrm{c}$. Six chambers of the Barrel and five of the End-Cap have been mounted in such a way to reproduce the first tracking tower at $\eta=0$ (Barrel), and a part of one octant of the three End-Cap tracking stations. Both systems were fully equipped with alignment sensors and temperature sensors. A dipole magnet allowed the beam to be steered across the chambers enabling the autocalibration of the r-t relation. The positions of the chambers as measured with the alignment system were compared with their positions measured by tracking high energy muons. Displacing one chamber from its original position enables a check of the alignment system. The middle chamber of the Barrel tower was displaced in steps of about $250 \mu \mathrm{m}$ up to $2 \mathrm{~mm}$ from its original position, in a direction forming an angle of $15^{\circ}$ with the normal to the beam direction. Fig 18 A) shows the correlation between the displacement measured with the alignment system and that measured by tracking, Fig. 18 B) shows the difference of the 
two measurements. The r.m.s. scatter of the two measurements is less than 20 $\mu \mathrm{m}$, demonstrating the correctness of the alignment concept in ATLAS.

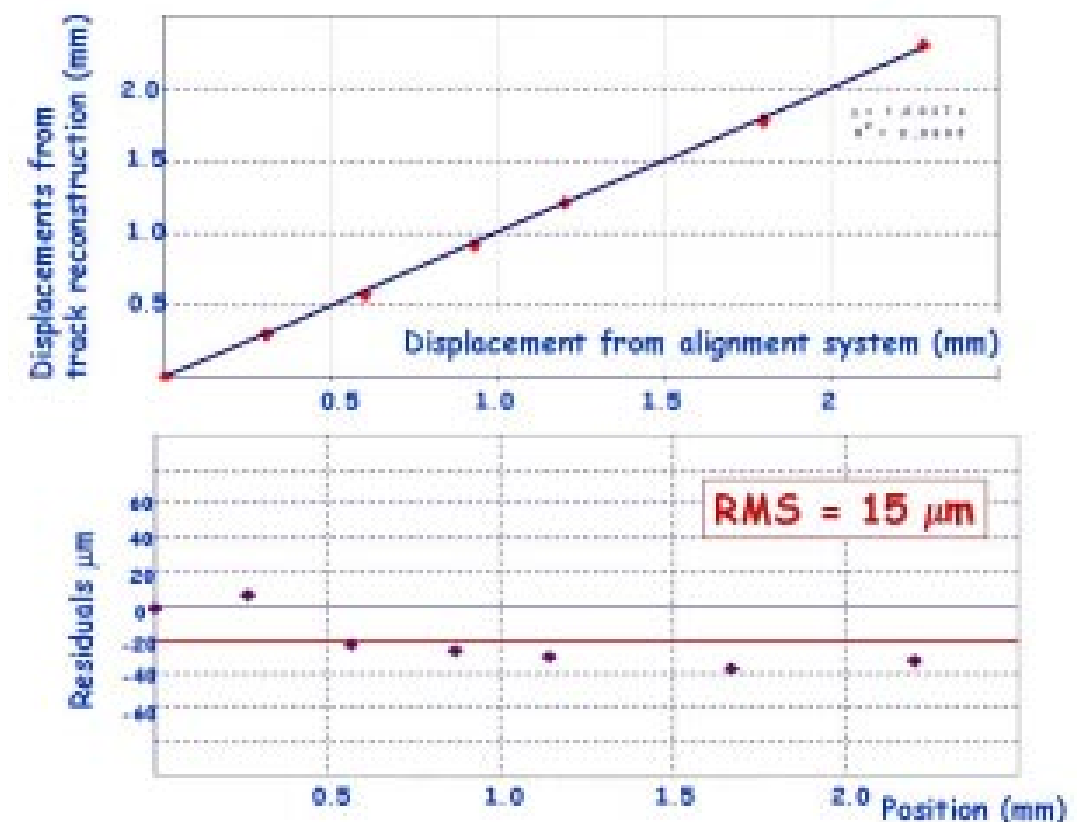

Fig. 18. A) Comparison of the displacement measured with the alignment system with that measured by tracking high energy muons in the detector. B) Difference of the two measurements, the r.m.s. scatter of the measured points is better than $20 \mu \mathrm{m}$.

\subsection{The Cathode Strip Chambers: CSC.}

The Cathode Strip Chambers are multiwire proportional chambers with cathode strip read-out. A schematic diagram of the Cathode Strip Chamber is shown in Fig. 19 [11].

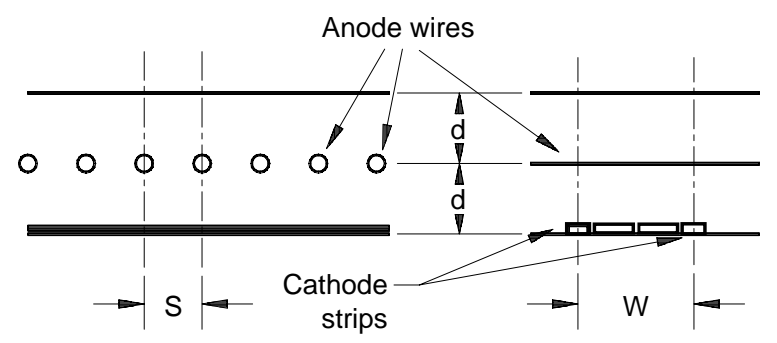

Fig. 19. Schematic diagram of the Cathode Strip Chambers.

The anode wires' pitch ( $\mathrm{S}$ in the figure) is $2.54 \mathrm{~mm}$ and the cathode readout pitch $(\mathrm{W})$ is $5.08 \mathrm{~mm}$. The cathode planes are equipped with orthogonal 
strips and the precision coordinate is obtained measuring the charge induced on the strips making the charge interpolation between neighboring strips. Typical resolution obtained with this read-out scheme is about $50 \mu \mathrm{m}$. The smallness of the basic cell implies also small maximum drift time (about $30 \mathrm{~ns}$ ) which is beneficial to keep the chamber occupancy low enough. The gas mixture used is based on $\mathrm{Ar}(30 \%), \mathrm{CO}_{2}(50 \%)$ and $\mathrm{CF}_{4}(20 \%)$, the wires are supplied by $2.6 \mathrm{kV}$, resulting in a gas gain of $10^{4}$. In ATLAS the CSCs will be arranged in two layers, each containing 4 layers of cells, enabling 8 high precision measured points on a single track. The shape of the chambers is trapezoidal and they will be mounted on the inner tracking wheel for $2<|\eta|<2.7$. In this region the counting rate due to photons and neutrons is of the order of $1 \mathrm{kHz} / \mathrm{cm}^{2}$, and this could cause a degradation of the measuring efficiency and/or resolution. Tests have been carried out at the GIF at CERN to study the dependence of efficiency and resolution on the counting rate. The results are presented in Fig. $20[12]$.

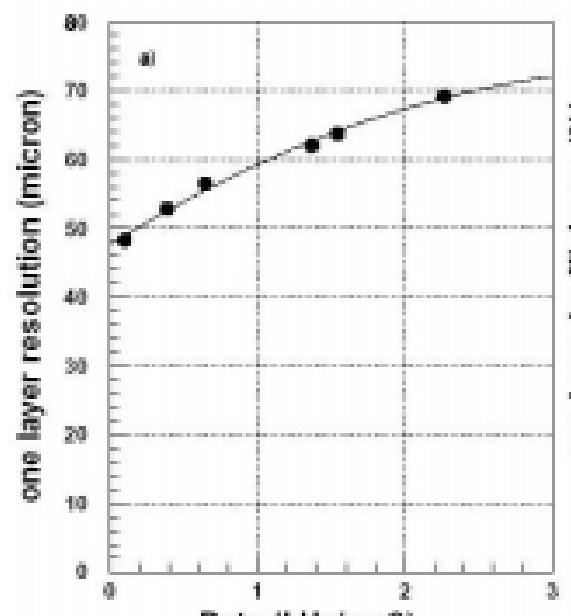

Rate $(\mathrm{kHz} / \mathrm{cm} 2)$

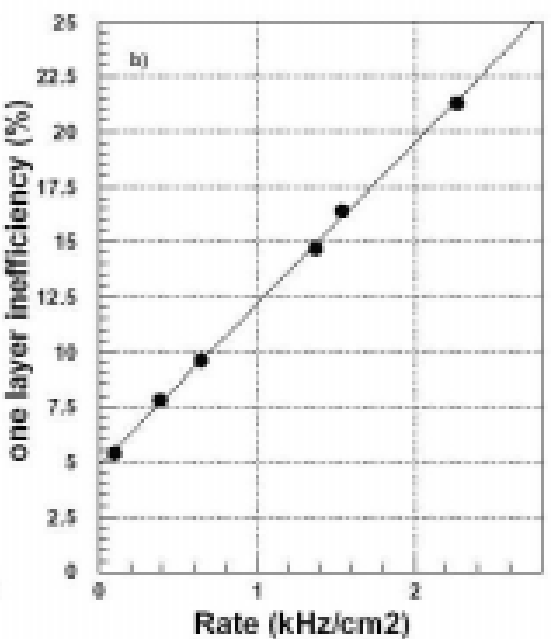

Rate (kHz/cm2)

Fig. 20. Single plane resolution and inefficiency as a function of the counting rate.

The single plane resolution degrades with the counting rate and, at about 2 $\mathrm{kHz} / \mathrm{cm}^{2}$, is about $70 \mu \mathrm{m}$. This effect is mainly due to overlapping signals that induce charge on the strips close to the muon track position. The same effects degrade the single wire efficiency, defined as the number of times a hit is closer to a track than $300 \mu \mathrm{m}$ with respect to all the reconstructed tracks (in this test, tracks were reconstructed with a silicon telescope). The inefficiency rises from $5 \%$ to $20 \%$ as a function of the rate. At the smaller rate the inefficiency is mainly due to the $\delta$-rays production on the chamber material. 


\section{Conclusion}

The ATLAS Muon Spectrometer is a large and complex device. It will be involved in many physics searches at the LHC, where it should provide both the trigger and the precise measurement of the muon momentum. Both the tracking and trigger chambers have been tested in test beams and proved to be adequate for the spectrometer requirements. System issues such as the alignment of the chambers were studied and tested at the H8 System Test and found to meet the specifications. In the next few years the focus will be on the integration of the trigger and tracking chambers with their control systems and the data acquisition as part of of the final installation in ATLAS.

\section{References}

1. ATLAS Technical Proposal, CERN/LHCC/94-43, LHCC/P2 15 December 1994.

2. ATLAS Muon Spectrometer TDR. CERN/LHCC/97-22, ATLAS TDR 10, 31 May 1997.

3. ATLAS Detector and Physics Performance TDR, CERN/LHCC/99-124 (1999).

4. P. Camarri et al.: RPCs for the ATLAS Level-1 muon trigger: Test beam results. ATL-MUON-98-234.

5. E. Duchovni et al.: Possible Utilization of Thin-Gap Chambers in the ATLAS Muon System; ATL-MUON-93-023.

6. C. Guyot et al.: Concepts for the mechanical realisation of the ATLAS muon Chamber alignment. ATL-MUON-94-062.

7. H. Groenstege.: The RASNIK/CCD 3D alignment system. ATL-MUON-94-063.

8. D. Drakoulakos et al.: XTOMO2, Stereo-Measurements of the MDT Muon Chambers using high precision X-ray Tomograph. ATLAS Note MUON-NO-155 (1997).

9. C. Bacci et al.: Autocalibration of High Precision Drift Tubes. Nucl. Phys. Proc. Suppl 54 (1997) 311.

10. M. Daile. Optimization and calibration of the Drift-Tube Chambers for the ATLAS Muon Spectrometer. Doctoral Thesis a the Ludwig-Maximilians-UniversitatMunchen (May 2000).

11. V. Polichronakos. A Proposal to Use the Cathode Strip Chambers (CSC) for the ATLAS Forward Muon System ATLAS-MUON-94-038.

12. A. Gordeev et Al.: CSC Performance at High Background Rates. ATL-MUON2000-005. 